\title{
Hiperplasia angiolinfoidea con eosinofilia: Presentación de un caso en cavidad oral y revisión de la literatura
}

\author{
O Iga Salinas $\mathrm{F}^{1 \mathrm{a}}$, Yamile Corredoira $\mathrm{S}^{2}$, G onzalo Rojas $\mathrm{A}^{3 \mathrm{a}}$. \\ Angiolymphoid hyperplasia of the lip \\ with eosinophilia. Report of one case
}

We report a 33 year-old female presenting with a $2 \mathrm{~cm}$ tumor of the upper lip lasting one year. The tumor was excised and the pathological examination showed multiple blood vessels with thickened walls, prominent endothelial cells, lymphoid follicles and an increased number of eosinophils. The final diagnosis was an angiolymphoid hyperplasia with eosinophilia. Six months after surgery, the patient was free of disease. This is a rare condition that must be distinguished from Kimura disease (Rev Méd Chile 2007; 135: 636-9).

(Key words: Angiolymphoid hyperplasia with eosinophilia; Kimura disease; Lip neoplasms)

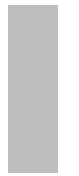

Recibido el 26 de abril, 2006. Aceptado el 16 de agosto, 2006.

${ }^{1}$ Centro de Referencia en Salud Cordillera, Santiago de Chile. ${ }^{2}$ Servicio de Anatomía Patológica del Hospital Santiago Oriente "Luis Tisné Brousse». Santiago de Chile. ${ }^{3}$ Servicio de Anatomía Patológica, Facultad de Odontología, Universidad de Chile. Santiago de Chile.

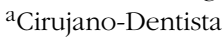

$\mathrm{L}$ a hiperplasia angiolinfoidea con eosinofilia (ALHE) es una lesión benigna de muy baja prevalencia. Se caracteriza por ser una condición vasculoproliferativa idiopática, de posible origen neoplásico ${ }^{1}$ y que se manifiesta como nódulos subcutáneos, ya sea múltiples o únicos, localizados habitualmente en la región de cabeza y cuello, en adultos jóvenes. Su ubicación en otras partes del cuerpo es más bien rara, pero se han descrito casos en manos $^{2}$ y pulmones $^{3}$, entre otras. Se han reportado casos que han aparecido durante el embarazo y por el uso de anticonceptivos orales ${ }^{4}$, por lo que se supone una dependencia hormonal. Un caso de ALHE fue comunicado

Correspondencia a: Gonzalo Rojas Alcayaga. Olivos 964, Independencia. Fono: 9781811. E mail: gorojas@uchile.cl en relación topográfica con un carcinoma de células escamosas de piel, que regresó espontáneamente una vez extirpada la neoplasia maligna. Esto supone la participación de factores angiogénicos en el desarrollo de ALHE ${ }^{5}$. Se caracteriza histológicamente por proliferación de vasos sanguíneos de paredes engrosadas tapizados por células endoteliales prominentes, infiltración del intersticio por células inflamatorias crónicas, principalmente eosinófilos y la presencia de folículos de células linfoides con centros germinativos ${ }^{4}$. Desde el punto de vista histológico, en algunos casos puede presentar características de neoplasias vasculares malignas ${ }^{6}$. Respecto al diagnóstico diferencial, la principal entidad que debe descartarse es la enfermedad de Kimura, pues ésta se asocia frecuentemente con falla renal ${ }^{7}$. El tratamiento de la ALHE es fundamentalmente quirúrgico ${ }^{2}$. 


\section{CaSo CLínICO}

Paciente mujer de 36 años que acudió a la Unidad Dental del Centro de Referencia de Salud de Peñalolén, Cordillera Oriente, Santiago, Chile. Consultó por aumento de volumen en labio superior lado izquierdo. La paciente señaló que la lesión había crecido lentamente desde aproximadamente 1 año desde que ella lo detectó. A la inspección clínica se observó el labio con aumento de volumen nodular, irregular, globuloso (como racimo), de aproximadamente $2 \mathrm{~cm}$ de diámetro mayor, indoloro, y de consistencia firme a la palpación, de límites más o menos difusos y recubierto por mucosa de aspecto normal (Figura 1). En la anamnesis no se pesquisaron otros procesos ni antecedentes mórbidos relevantes.

Con la hipótesis diagnóstica de adenoma pleomorfo, se procedió a realizar la biopsia excisional bajo anestesia local. Se logró extraer toda la tumoración además de tejido adyacente, ya que si bien se observaba algo de fibrosis periférica, ésta se entremezclaba con el tejido conjuntivo y capa muscular de los labios. La muestra fue enviada a análisis histopatológico.

El informe histopatológico reveló la presencia de proliferación bien delimitada, pero no capsula$\mathrm{da}$, de un tejido compuesto por vasos sanguíneos de paredes engrosadas, entremezclados con un infiltrado linfocitario que se disponía a manera de folículos con centros germinativos, además de presencia destacada de eosinófilos (Figura 2). El tejido presentaba múltiples tabicaciones por haces de fibras colágenas. El diagnóstico histopatológico correspondió a hiperplasia angiolinfoidea con eosinofilia. Los valores de los distintos parámetros del hemograma estaban dentro de los rangos normales.

La paciente fue controlada a los 10 días y se procedió al retiro de la sutura. Luego se volvió a controlar al mes y 6 meses de realizada la cirugía, sin observarse recidiva.

\section{Comentarios}

La hiperplasia angiolinfoidea con eosinofilia (ALHE), es una entidad patológica de baja prevalencia y que en algunas ocasiones se presenta en los tejidos blandos de la cavidad bucal. El caso que se presenta cumple con las características habitualmente descritas para esta lesión. En esa ubicación anatómica, las lesiones de mayor prevalencia son las neoplasias benignas de glándulas salivales, principalmente el adenoma pleomorfo ${ }^{8}$. Neoplasias benignas de otros tejidos tales como neurilemoma, neurofibromas, leiomioma y lipomas, entre otras, han sido descritas en ubicación labial superior ${ }^{8}$. También se han reportado casos en los que el diagnóstico diferencial ha considera-

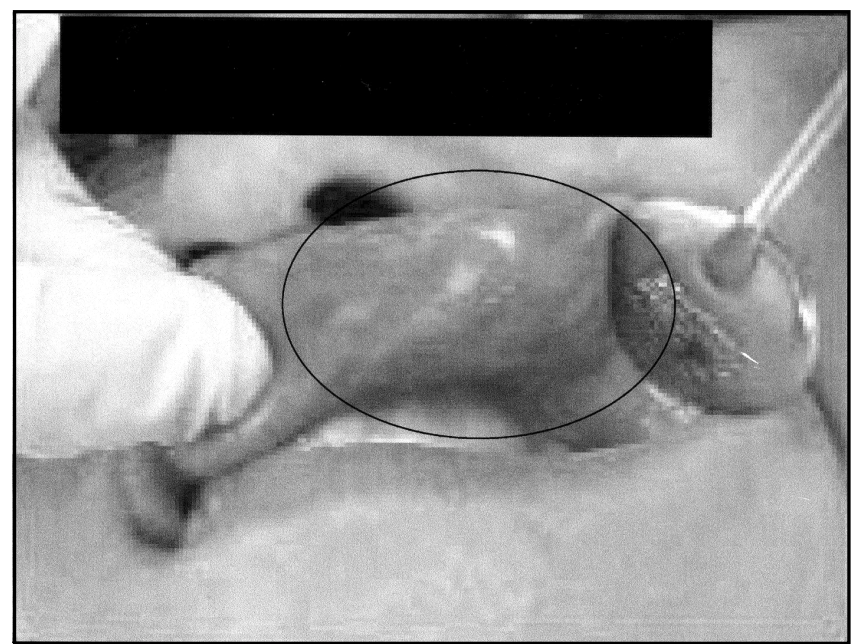

Figura 1. Se aprecia la lesión nodular ubicada en labio superior lado izquierdo (encerrada en círculo). 


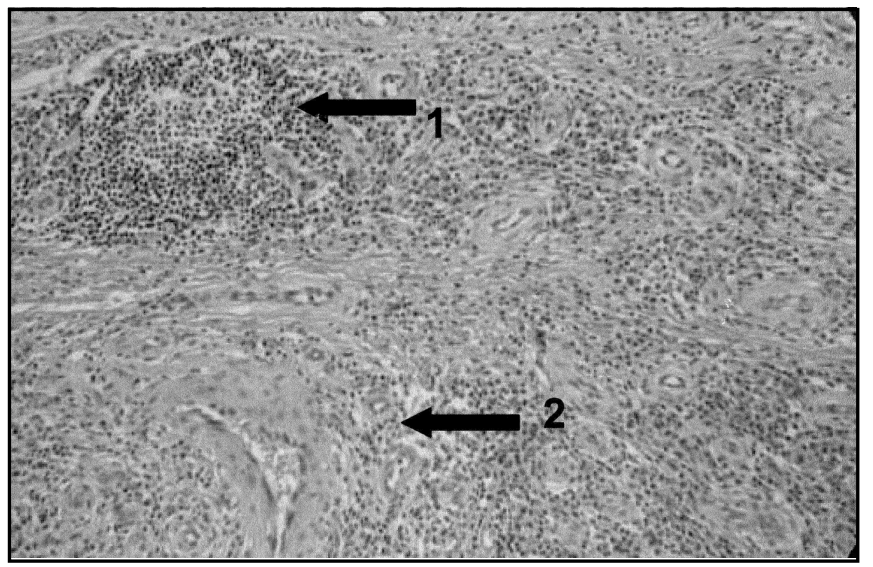

Figura 2. Imagen histopatológica. Se observa la presencia de folículos linfoideos con centros germinativos (señalado por flecha 1), algunos vasos sanguíneos de paredes engrosadas (señalado por flecha 2) y tabicaciones de colágeno (aumento 10x, tinción hematoxilina-eosina).

do neoplasias malignas, como angiosarcomas ${ }^{6}$. En el caso expuesto, las características clínicas eran compatibles con adenoma pleomorfo.

Otros casos comunicados de ALHE en cavidad oral son escasos. Suzuki y cols ${ }^{9}$ también publicaron un caso de ubicación en labio superior con características similares al caso nuestro. Otro caso, en lengua, fue reportado por Pack y cols ${ }^{10}$, ubicación aún más infrecuente, sin embargo, y a diferencia del caso presentado, la lesión lingual se presentó como una úlcera dolorosa. En ambos casos el diagnóstico fue histopatológico y los hallazgos principales fueron la proliferación de vasos sanguíneos de pequeño calibre con endoteliocitos prominentes, además de un infiltrado inflamatorio compuesto principalmente de linfocitos y eosinófilos. Sin embargo, en ellos no se observó la formación de folículos linfoideos, aspecto que sí fue observado en nuestro caso.

La mayor complejidad en el diagnóstico de la ALHE es la diferenciación con la enfermedad de Kimura (EK). De hecho, existen similitudes tanto clínicas como histopatológicas, incluso se ha planteado que ambas entidades patológicas pueden representar variaciones de una misma enfer$\operatorname{medad}^{11}$. Obviamente, lesiones compatibles con enfermedad de Kimura también se presentan en cavidad bucal ${ }^{12}$. Sin embargo, existen ciertas características que permiten diferenciarlas. La edad y el sexo de la paciente coinciden más con ALHE, además, la paciente no presentaba ni compromiso ganglionar regional ni patología renal, situación más relacionada con EK. La extensión hacia planos musculares, la gran proliferación vascular y la presencia de células endoteliales altas observada histológicamente en este caso, es característica de la ALHE ${ }^{1,7,13}$.

La eosinofilia, situación habitual en la EK, no se presentó en el caso expuesto, lo que se condice con el diagnóstico de ALHE.

Es importante el correcto diagnóstico de la ALHE, en parte por su significativo porcentaje de recidiva, lo que amerita seguimiento en el tiempo y porque si bien su tratamiento es eminentemente quirúrgico en algunas ocasiones es posible de ser complementado por tratamientos farmacológicos con corticoides, retinoides orales, inmunomoduladores como el imiquimod ${ }^{14}$ y agentes antialérgi$\cos ^{15}$. La radioterapia también ha sido utilizada con bastante éxito ${ }^{16}$. En el caso presentado y después de seis meses no se ha registrado indicio alguno de recidiva, por lo que se supone que el tratamiento quirúrgico fue suficiente. 


\section{Referencias}

1. SEREGARD S. Angiolymphoid hyperplasia with eosinophilia should not be confused with Kimura's disease. Acta Ophthalmol Scand 2001; 79: 91-3.

2. Satpathy A, Moss C, Raafat F, Slator R. Spontaneous regression of a rare tumour in a Child: Angiolymphoid Hyperplasia with Eosinophilia of the Hand, Case Report an Review of the Literature. Br J Plast Surg 2005; 58: 865-8.

3. Morán C A, Suster S. Angiolymphoid hyperplasia with eosinophilia (epithelioid hemangioma) of the lung: a clinicopathologic and immunohistochemical study of two cases. Am J Clin Pathol 2005; 123: 762-5.

4. Zarrin-Khameh N, Spoden J, Tran R. Angiolymphoid Hyperplasia with Eosinophilia Associated with Pregnancy. Arch Pathol Lab Med 2005; 129: 1168-71.

5. Lugo-Janer A, Montalvo L, Ruiz-Santiago H, SánCHEz JL. Angiolymphoid hyperplasia with eosinophilia associated to a squamous cell carcinoma of the ear. Dermatol Surg 2005; 129: 1168-71.

6. Zettouni N, Hanna S, Loree TR, Brooks J, Cheney R. Angiolymphoid Hyperplasia with Eosinophilia: A Classic Clinical Presentation With Histologic Features of Angiosarcoma. Dermatol Surg 2002; 28: 772.

7. Ramchandani Pl, Sabesan T, Hussein K. Angiolymphoid hyperplasia with eosinophilia masquerading as Kimura disease. Br J Oral Maxillofac Surg 2005; 43: 249-52.

8. Neville B, Damm D, Allen C, Bouquot J. Salivary Gland Pathology and Soft Tissue Tumors. En:
Oral and Maxillofacial Pathology. Segunda Edición. Philadelphia. USA. Saunders Company. 2002; 406-78.

9. Suzuki H, Hatamochi A, Horie M, Suzuki T, Yamazaki S. A case of angiolymphoid hyperplasia with eosinophilia (ALHE) of the upper lip. J Dermatol 2005; 32: 991-5.

10. Park Y, Chung J, Cho CG. Angiolymphoid hyperplasia with esoinophilia of the tongue: report of a case and review of the literature. Oral Oncol 2002; 38: 103-6.

11. Chong WS, Thomas A, Goh CL. Kimura's disease and angiolymphoid hyperplasia with eosinophilia: two disease entities in the same patient: case report and review of the literature. Int J Dermatol 2006; 45: 139-45.

12. Terakado N, Sasaki A, Takebayashi T, Matsumura T, Kojou T. A case of Kimura's disease of the hard palate. Int J Oral Maxillofac Surg 2002; 31: 222-4.

13. Asadi AK. Angiolymphoid hyperplasia with eosinophilia. Dermatol Online J 2002; 8: 10.

14. Redondo P, Del Olmo J, Idoate M. Angiolymphoid hyperplasia with eosinophilia successfully treated with imiquimod. Br J Dermatol 2004; 151: 1110.

15. Harada K, Kambe Y, Takeda H, Nakano H, Hanada K. Angiolymphoid hyperplasia with eosinophilia: successful treatment with the antiallergic agent suplatast tosilate. Dermatology 2004; 208: 176-7.

16. Conill C, Toscas I, Mascaró JM Jr, Vilalta A, MASCARÓ JM. Angiolymphoid hyperplasia with eosinophilia of the nail bed and bone: successful treatment with radiation therapy. J Eur Acad Dermatol Venereol 2004; 18: 584-5. 